\title{
Broadband Suppression Properties of Active Leaking Carrier Cancellers
}

\author{
Gregor Lasser, Robert Langwieser, Arpad L. Scholtz \\ Vienna University of Technology \\ Institute of Communications and Radio-Frequency Engineering \\ Gusshausstrasse 25/389, 1040 Vienna, Austria \\ Email: gregor.lasser@nt.tuwien.ac.at
}

\begin{abstract}
In passive UHF RFID systems backscattering is used for tag to reader communication. This technique relies on a continuous wave signal being transmitted by the reader during the tag's data transfer. In order to separate transmission and reception paths circulators, directional couplers or disjoint transmit and receive antennas can be used. Perfect isolation is not achievable with any of those approaches. So a leaking carrier is present at the receiver in any case. It is possible to reduce the interference from this signal by some kind of leaking carrier cancellation. Such a cancellation prevents receiver blocking and reduces the baseband hardware's requirements, depending on the receiver concept. Usually, the narrowband properties of carrier cancellers are studied. This is only sufficient for conventional RFID systems, if the transmitter noise can be neglected. Broadband RFID systems, as recently discussed in literature, also require broadband leaking carrier cancellers. In this paper the broadband suppression properties of carrier cancellers are first investigated theoretically. Further a hardware implementation is presented and characterized. Finally, measurement results are compared to the theoretical findings.
\end{abstract}

\section{INTRODUCTION}

Passive UHF RFID tags require a continuous wave signal from a reader to supply their internal circuitry and enable backscattering. This carrier signal is also present during tag to reader communication events. The backscattering technique used for data transfer implies a tag signal at the same carrier frequency.

For transmission and reception path separation two main techniques exist. The first one uses disjoint transmit and receive antennas placed suitably apart from each other, the second one utilizes a single antenna and a device that separates forward and backward wave components. This device can be either a circulator or a directional coupler. However, in both approaches it is hard to push the isolation between transmitter and receiver above $20 \mathrm{~dB}$. This holds particularly in non static scenarios with moving scatterers.

Many high performance RFID readers employ one of several possible realizations of active interference reduction systems to avoid large carrier amplitudes at the receiver input caused by insufficient receiver-transmitter isolation [1], [2], [3]. This is important because the leaking carrier not only

This work has been funded by Infineon Technologies Austria AG and the Christian Doppler Laboratory for Design Methodology of Signal Processing Algorithms. changes the modulation properties of the received signal, it can also cause receiver blocking or even worse, destruction of the receiver. Active interference reduction is a well known technique also called leaking carrier cancellation [1], carrier compensation or direct coupling compensation [2] and is also used in radar applications [4].

A setup of an RFID reader with leaking carrier canceller is shown in Fig. 1. The idea is to cancel the leaking carrier by generating its inverse and then summing it with the received signal. In the context of carrier compensation a signal's inverse is its additive inverse often also called inverted signal. To achieve this, the transmitter is connected to a directional coupler which is used to extract a sample of the transmit signal for the cancelling circuitry. The main output of this coupler is fed to the transmit antenna in two antenna systems, or to a circulator in one antenna systems as illustrated in Fig. 1, to separate transmission and reception paths. The receiver antenna or the receiver output port of the circulator is not directly connected to the receiver, the received signal is routed through a second directional coupler instead. Here the compensation path, which is composed of a vector modulator and an amplifier, merges with the main receiving path. The reconstructed inverse leaking carrier is added to the received signal resulting in a purified received signal that is fed to the receiver.

\section{Motivation FOR BROADBAND SUPPRESSION}

The usual approach to characterize leaking carrier cancellers in RFID systems is to measure the attenuation realized at the reader's carrier frequency. This is only sufficient for an ideal RFID system, because here broadband interference cancellation is not required. During reception the only possible interferer is the reader's unmodulated continuous wave carrier. Obviously only a narrowband canceller is necessary to suppress this sine wave.

For real systems the situation gets different, when noise has to be taken into account. The transmit signal will contain noise components, depending on the quality of the transmitter's oscillators and amplifiers. Here broadband suppression becomes an issue, otherwise the noise components of the leaking signal and the cancellation signal may even interfere constructively. 


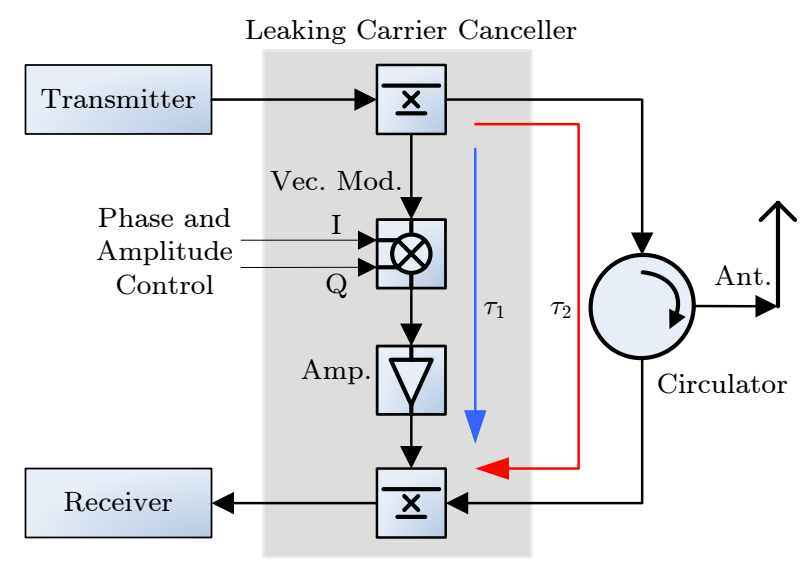

Fig. 1. RFID system with Leaking Carrier Canceller

Current RFID tags require not more than $5 \mu \mathrm{W}$ for operation [1]. Under legal limit conditions with $2 \mathrm{~W} \mathrm{ERP}^{1}$ transmitted by the reader, a receiver input signal of $-80 \mathrm{dBm}$ can be expected at maximum operating distance, depending on the antennas used [5]. When $20 \mathrm{~dB}$ of intrinsic transmitter receiver isolation is assumed, the signal of interest is $93 \mathrm{~dB}$ below the interfering carrier, so the carrier's phase noise is critical. With proper broadband interference cancellation not only the carrier but also its phase noise is considerably reduced at the receiver input.

A rather new research field is to combine RFID systems with spread spectrum technology. While some authors suggest to use spread spectrum transmission in the uplink from tag to reader [6], others investigate to use this technology at the transmitter to cope with dense reader environments [7]. In this scenario spreading modulation is also present at the transmitter during tag to reader communication events. To operate a leaking carrier canceller under such conditions it is absolutely necessary that this canceller is optimized for broadband suppression to achieve satisfactory results.

\section{ANALYTICAL MODELING}

To model the behavior of active leaking carrier cancellers a single point of reflection which causes the interference is assumed. This could be, for instance, an imperfect circulator (see Fig. 1). All other components like cables or the antenna are perfectly matched and therefore do not produce reflections which end up in the receiver. We also assume that the unwanted reflection in the circulator does not introduce any phase shifts in the frequency band of interest. The directional coupler that is used to combine the receiving path with the compensation path is modeled as an ideal summation device.

With these assumptions the impulse response $h(t)$ of the overall system between the transmitter output port and the receiver input can be derived:

$$
h(t)=A \cdot \delta\left(t-\tau_{2}\right)-B \cdot \delta\left(t-\tau_{1}\right) * p(t)
$$

\footnotetext{
${ }^{1}$ For UHF RFID applications $2 \mathrm{~W}$ ERP is the maximum allowed transmission power in most European states.
}

Here $\delta(t)$ is the Dirac-function and $p(t)$ is the impulse response of the vector modulator. The first term describes the unwanted signal component with amplitude $A$ and the second one the component produced by the canceller, scaled by $B$. With reference to the transmitter, $\tau_{2}$ and $\tau_{1}$ are the delays of those components at the receiver. It is obvious that total suppression requires the amplitudes of both terms to be equal, so we set $A=B=1$ and $|p(t)|=1$. For convenience we introduce $\Delta \tau=\tau_{1}-\tau_{2}$ and substitute $\tau_{1}$. Without loss of generality, $\tau_{2}$ can be set to zero, so that $\tau_{1}$ becomes $\Delta \tau$.

The vector modulator's impulse response $p(t)$ can be expressed as

$$
p(t)=\cos (\alpha) \delta(t)+\sin (\alpha) \frac{1}{\pi t}
$$

so that $\cos (\alpha)$ represents the amplitude of the inphase component and $\sin (\alpha)$ the quadrature component's amplitude which vectorially sum up to 1 . The phase shift introduced by the vector modulator is represented by the angle $\alpha$. The quadrature component is generated by the term $1 / \pi t$, the impulse response which corresponds to a Hilbert transform.

Insertion of (2) into (1) and adopting the described simplifications leads to the system's over-all impulse response:

$$
h(t)=\delta(t)-\left(\cos (\alpha) \delta(t-\Delta \tau)+\sin (\alpha) \delta(t-\Delta \tau) * \frac{1}{\pi t}\right)
$$

The system's transfer function $H(j \omega)$ is found by Fourier transformation:

$$
\begin{aligned}
H(j \omega) & =\mathcal{F}\{h(t)\} \\
& =1-\left(\cos (\alpha) e^{-j \omega \Delta \tau}+\sin (\alpha) e^{-j \omega \Delta \tau}(-j \cdot \operatorname{sign}(\omega))\right)
\end{aligned}
$$

To investigate the amount of carrier suppression the absolute value is calculated.

$$
|H(j \omega)|^{2}=2(1-\cos (\omega \Delta \tau+\alpha \cdot \operatorname{sign}(\omega)))
$$

For nonnegative values of $\omega$ the signum function in (5) can be omitted:

$$
|H(j \omega)|^{2}=2(1-\cos (\omega \Delta \tau+\alpha)) \quad \mid \omega \geq 0
$$

The absolute value of the transfer function (6) is a periodic function in $\omega$. Fig. 2 depicts a plot of (6) with $\Delta \tau=1.061 \mathrm{~ns}$ and $\alpha=29.02^{\circ}$. As indicated in Fig. 2 we define an attenuation bandwidth $B=f_{u}-f_{l}$ where the level of suppression is above the desired attenuation $D$. With increasing delay offset $\Delta \tau$ of the interference and the compensation paths, the period of $|H(j \omega)|^{2}$ shortens and reduces the bandwidth $B$ for a specified suppression value.

To calculate the maximum allowed delay offset $\Delta \tau$ for specific values of $B$ and $D$ we set $\omega=2 \pi f_{u}$ :

$$
2\left(1-\cos \left(2 \pi f_{u} \Delta \tau+\alpha\right)\right)=D
$$

To achieve maximum extinction at the frequency $f_{0}$ we find the constraint

$$
2 \pi f_{0} \Delta \tau+\alpha=2 \pi n, n \in \mathbb{Z}
$$




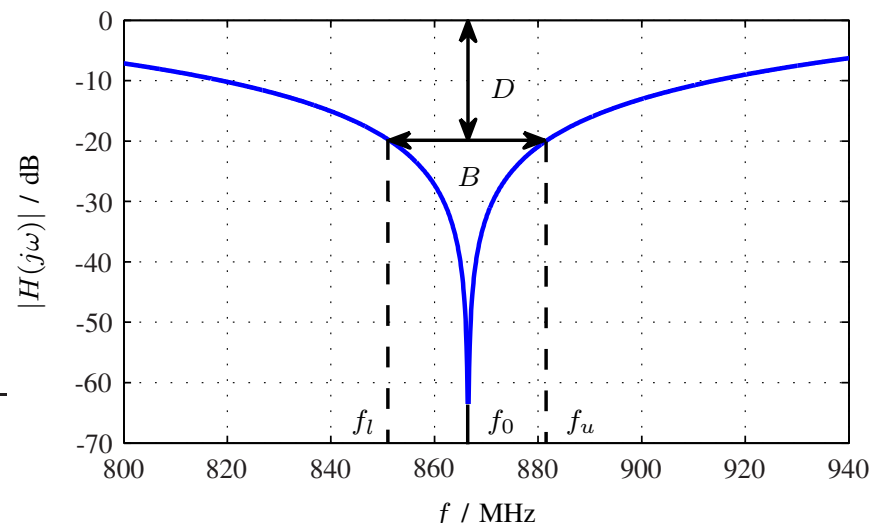

Fig. 2. Transfer function of the modeled suppression system with $\Delta \tau=1.061 \mathrm{~ns}$ and $\alpha=29.02^{\circ}$

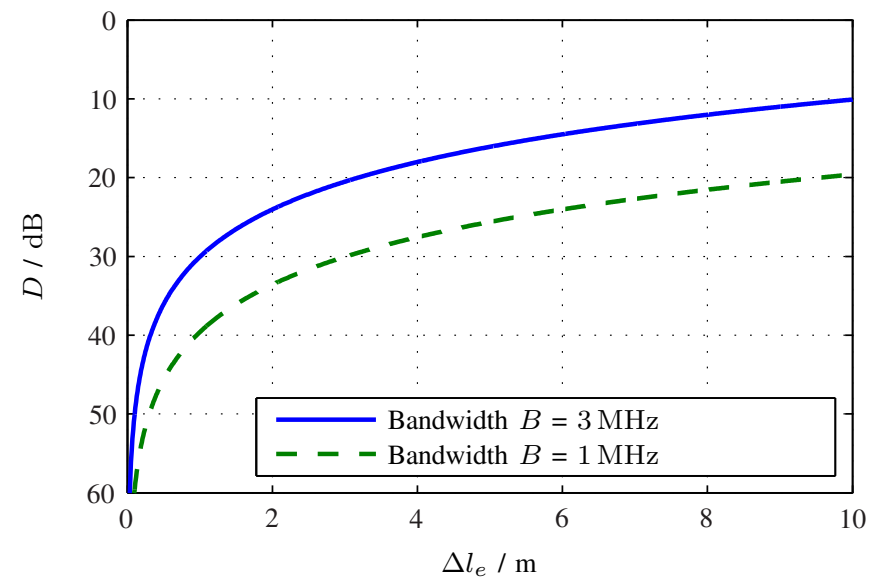

Fig. 3. Attenuation plotted over the electrical offset length $\Delta l_{e}$ for two fixed bandwidth values

When we use (8) to express $\alpha$ and insert this expression in (7) we get

$$
\Delta \tau=\frac{\arccos \left(1-\frac{D}{2}\right)}{\pi B}
$$

where $f_{u}-f_{0}=B / 2$ was used.

For interpretation it might be more intuitive to convert the delay offset $\Delta \tau$ into an electrical offset length $\Delta l_{e}=\Delta \tau \cdot c_{0}$. In Fig. 3 the achieved attenuation is plotted over $\Delta l_{e}$ for two different attenuation bandwidth values. The maximum allowed difference in electrical length for $40 \mathrm{~dB}$ attenuation over the full European RFID band, which has a bandwidth of $3 \mathrm{MHz}$, is $318 \mathrm{~mm}$.

When the leaking carrier canceller is used to compensate for an imperfect circulator in a single antenna scenario, the $318 \mathrm{~mm}$ boundary is easily achievable. The broadband suppression of reflections caused by badly matched antennas is more challenging. Especially, for non-handheld RFID readers where the antenna is attached with a cable of arbitrary length, the suppression bandwidth or rather the broadband attenuation can be severely degraded when the compensation path length is not adjusted accordingly.

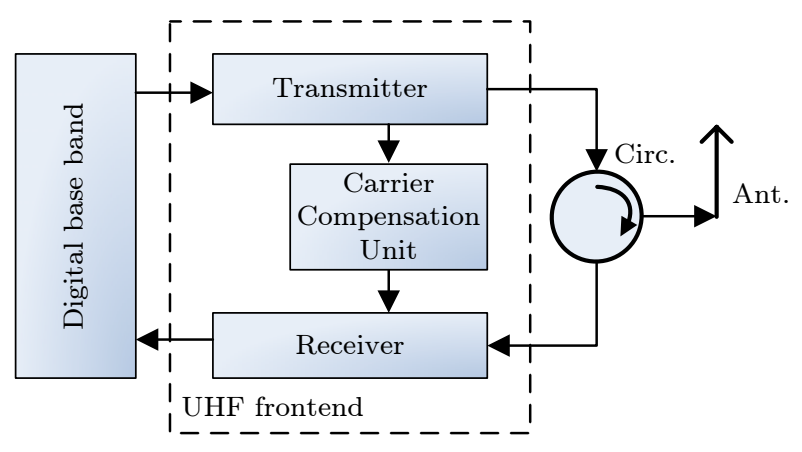

Fig. 4. Block diagram of the RFID testbed

\section{HARDWARE REALIZATION}

The hardware presented in the following section has been developed to enhance the capabilities of a new RFID testbed frontend designed at our institute [8]. Fig. 4 gives an overview of the modules of this testbed. The leaking carrier canceller's pick-up coupler and insertion coupler were implemented at the transmitter and the receiver, accordingly. The core of the carrier canceller, a vector modulator and an amplifier, were implemented as a separate assembly called Carrier Compensation Unit (CCU). A picture of the CCU is printed in Fig. 5.

The CCU's vector modulator functionality is provided by an IC from Analog Devices, the AD8340. This IC splits up the input signal in an inphase and a quadrature component with an integrated polyphase network. Further, this device contains separate balanced variable attenuators for the I and Q channel. These internal balanced attenuators allow to change the corresponding channel's polarity, so that a true $360^{\circ}$ phase shift range can be obtained. The supported amplitude range is $30 \mathrm{~dB}$. To compensate the coupler losses (at the transmitter as well as at the receiver) and enable cancellation of strong leaking carrier signals, the vector modulator is followed by an amplifier with a high $1 \mathrm{~dB}$ compression point of $27 \mathrm{dBm}$.

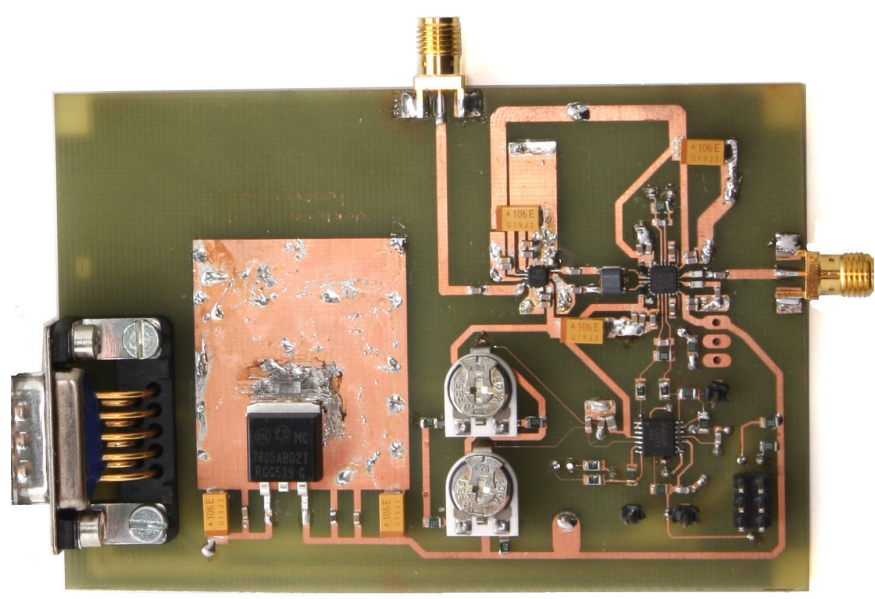

Fig. 5. Picture of the CCU module 


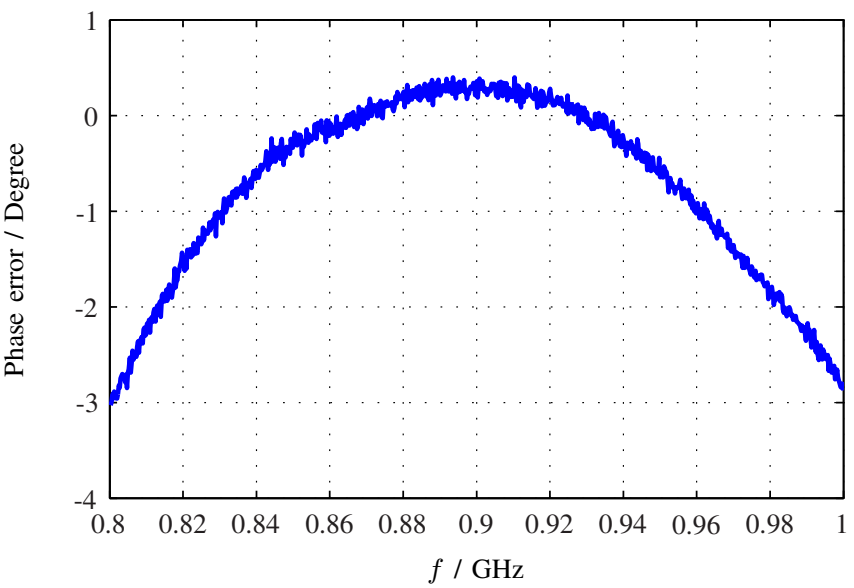

Fig. 6. Relative phase error of the $\mathrm{CCU}$

\section{Measurement Results}

Two types of measurements were carried out to characterize the CCU. They are presented in the following subsections. In the first one, a vector network analyzer was used to measure the CCU's phase response. In the second one the broadband suppression performance of the $\mathrm{CCU}$ was analyzed and the carrier suppression capabilities were verified.

\section{A. Phase Error}

To explore the possibilities of broad-band compensation, the relative phase error of the CCU was measured. Here the phase error is defined as the deviation from an ideal linear phase response. Fig. 6 represents the phase offset with reference to $866 \mathrm{MHz}$ measured between input and output port of the CCU. The phase error is smaller than $3^{\circ}$ over a frequency range from 800 to $1000 \mathrm{MHz}$. Over the UHF RFID band the phase error is almost negligible.

\section{B. Broadband Suppression Performance}

1) Measurement Setup: To measure the realizable suppression ratio the test setup presented in Fig. 7 was used. The vector network analyzer available provides direct access to the source ports of the internal measurement coupler. This access was used to insert a resistive $6 \mathrm{~dB}$-power splitter to provide a reference signal for the CCU input port. Losses of the network analyzer's test set were compensated in the source path. Ports one and two of the network analyzer were connected to the main path of a directional coupler, which was used to insert the output of the CCU module. A variable delay line was placed in the compensation path between power splitter and $\mathrm{CCU}$ to enable path length adaption. During the measurement campaign different cables of fixed length were inserted in the reference path to generate defined path length offsets.

The network analyzer was calibrated using a regular SOLT (Short Open Load Thru) method, but taking the terminated directional coupler as "Thru".

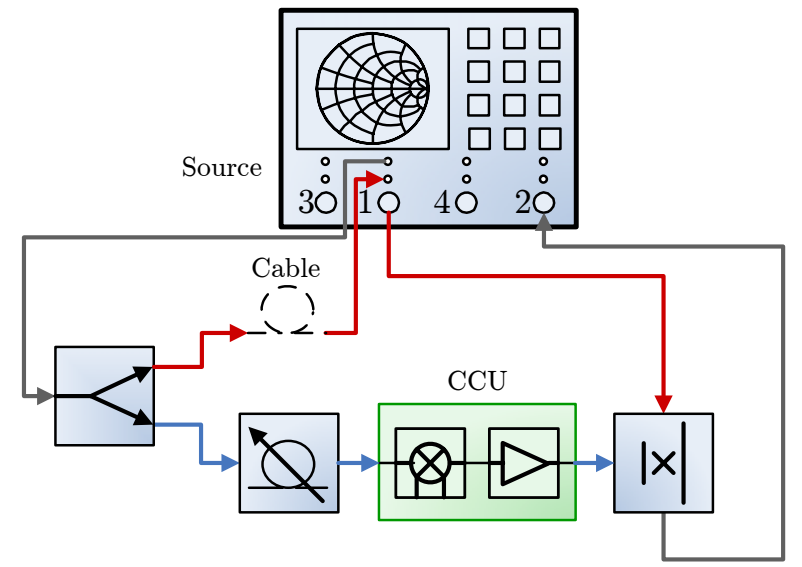

Fig. 7. Measurement setup for broadband suppression properties of the $\mathrm{CCU}$ as leaking carrier canceller

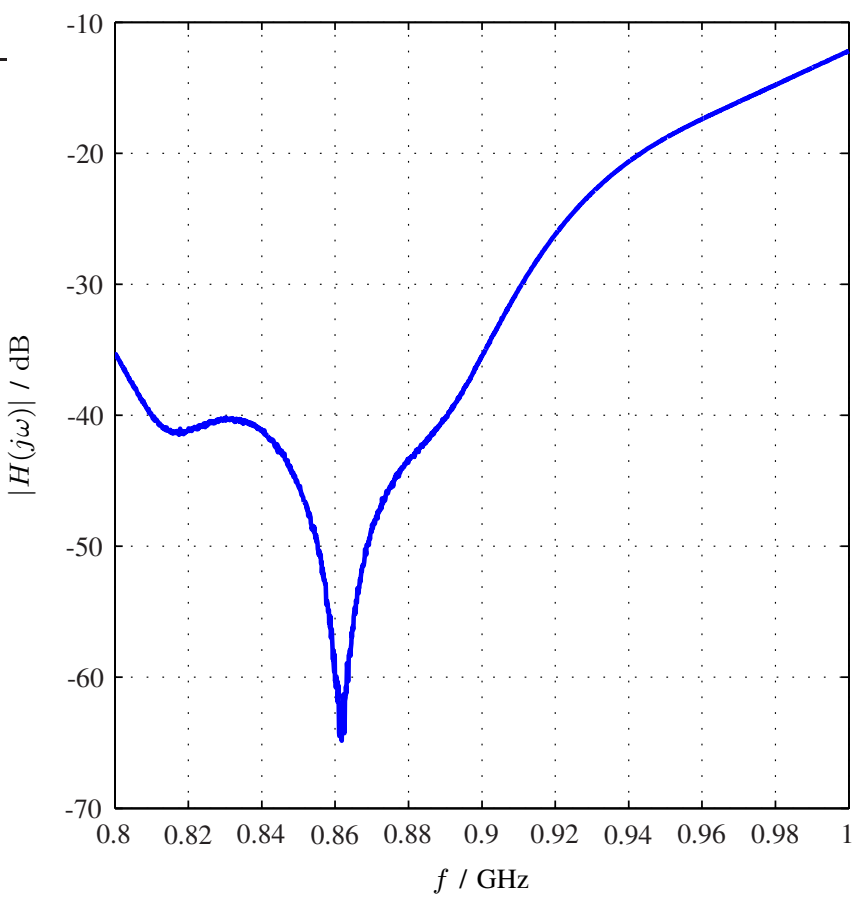

Fig. 8. Magnitude response of the measurement test setup with optimized reference path delay

2) Suppression Performance: In a first step the electrical path lengths of the main and compensation path were matched. This was done by continuous adjustments of the CCU's vector modulator control input voltages and changing the setting of the variable delay line until no further enhancement of the attenuation bandwidth could be achieved. For this adjustment a target suppression $D$ of $40 \mathrm{~dB}$ was chosen. Fig. 8 presents the measurement results of the leaking carrier canceller's magnitude response at this setting. A $40 \mathrm{~dB}$ suppression bandwidth $B$ of $82 \mathrm{MHz}$ could be achieved. The dispersion of CCU, cables and directional coupler prohibit further enhancement of this value. This first measurement serves as a reference for the following measurements, especially because for frequencies 


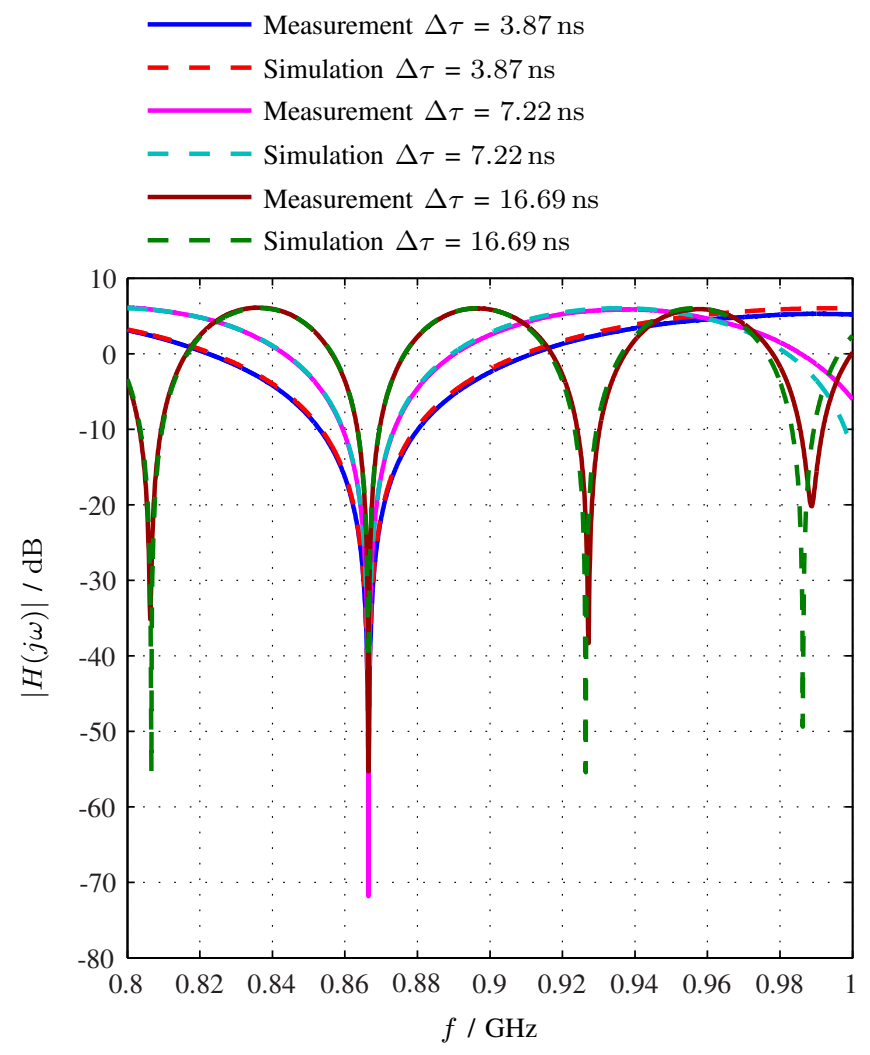

Fig. 9. Comparison of measured and simulated magnitude responses for different path delay offsets

around $866 \mathrm{MHz}$ the offset delay $\Delta \tau$ can be assumed to be zero.

In a second step fixed delays were introduced in the reference path by means of coaxial cables. These cables were characterized for their electrical length in a separate measurement with a conventionally calibrated network analyzer. Cables with electrical lengths of $3.87 \mathrm{~ns}, 7.22 \mathrm{~ns}$ and $16.69 \mathrm{~ns}$ were used. In our case this corresponds to mechanical lengths of $82 \mathrm{~cm}, 152 \mathrm{~cm}$ and $353 \mathrm{~cm}$. For every measurement the phase controls of the CCU were adjusted for maximum attenuation at $866.5 \mathrm{MHz}$. The measured magnitude response is plotted in Fig. 9. At the center frequency of $866.5 \mathrm{MHz}$ an attenuation between $55 \mathrm{~dB}$ and $70 \mathrm{~dB}$ was realized.
The dashed lines correspond to simulations according to (6), where the measured values of the electrical length of the three test cables were used as $\Delta \tau$. Measured and simulated curves show strong conformity, only at frequencies above $980 \mathrm{MHz}$ a slight deviation can be noticed.

\section{CONCLUSION}

To enhance the performance of RFID readers with a leaking carrier canceller not only the carrier, but also the noise produced by the reader has to be taken into account. The critical point is that the suppression bandwidth realized with the carrier canceller must be broad enough to capture all noise components of the transmitter in the reception bandwidth. In this paper an analytical model for RFID systems with a leaking carrier canceller and one dominant reflector was derived. This model was used to calculate the maximum allowed delay offset between the main path and the compensation path to achieve a certain suppression bandwidth.

A hardware realization of a leaking carrier canceller and measurement results were presented. In a test setup a $40 \mathrm{~dB}$ attenuation bandwidth of $82 \mathrm{MHz}$ could be achieved. The measurement curves show good accordance to the simulated curves of the analytical model derived in this paper.

\section{REFERENCES}

[1] Y. Liu, Q. Zhang, and M. Zheng, "Signal analysis and design criteria for UHF RFID reader,” ITS Telecommunications Proceedings, pp. 233-236, June 2006.

[2] J.-P. Curty, M. Declercq, C. Dehollain, and N. Joehl, Design and Optimization of Passive UHF RFID Systems, 1st ed. Springer, 2007.

[3] T. I. Al-Mahdawi, "Adaptive coherent RFID reader carrier cancellation," U.S. patent US 2006/0183454, filed Aug. 17, 2006.

[4] P. Beasley, A. Stove, B. Reits, and B. As, "Solving the problems of a single antenna frequency modulated CW radar," Record of the IEEE 1990 International Radar Conference, pp. 391 - 395, May 1990.

[5] G. Lasser, "Development of a UHF frontend for a comprehensive RFID rapid prototyping system," Master's thesis, Vienna University of Technology, October 2008.

[6] G. Mazurek, "Design of RFID system with DS-CDMA transmission," in 4th IEEE Conference on Automation Science and Engineering, Key Bridge Marriott, Washington DC, USA, August 2008.

[7] H. Deng, "Performance enhancement of radio frequency identification (RFID) using spread spectrum technology," Antennas and Propagation Society International Symposium, pp. 1205 - 1208, June 2007.

[8] R. Langwieser, G. Lasser, C. Angerer, M. Rupp, and A. L. Scholtz, "A modular UHF reader frontend for a flexible RFID testbed," in The 2nd Int. EURASIP Workshop on RFID Technology, Budapest, Hungary, July 2008. 